\title{
Timeliness of care in patients with lung cancer: a systematic review
}

\author{
J K Olsson, ${ }^{1,2}$ E M Schultz, ${ }^{2}$ M K Gould ${ }^{1,2}$
}

- A table of the studies included in the analysis is published online only at http:// thorax.bmj.com/content/vol64/ issueg

${ }^{1}$ VA Palo Alto Health Care System, Palo Alto, California, USA:

2 Stanford School of Medicine,

Stanford, California, USA

Correspondence to:

Dr M K Gould, VA Palo Alto

Health Care System, 3801

Miranda Ave (111P). Palo Alto,

CA 94304, USA; gould@

stanford.edu

Received 17 October 2008 Accepted 30 March 2009

\begin{abstract}
Background: Timeliness is an important dimension of health care quality. It is unclear whether timeliness improves clinical outcomes in patients with lung cancer. Methods: This study systematically reviewed studies that described timeliness of care, examined associations between timeliness and clinical outcomes or tested an intervention to improve timeliness of care in patients with lung cancer. English language studies published between 1 January 1995 and 1 June 2007 were included. Two reviewers independently abstracted data on study methods, population, sample size, relevant time intervals and outcomes.
\end{abstract}

Results: 49 studies were identified that reported at least one time interval in lung cancer care, 18 studies that examined the association between timeliness and clinical outcomes and 8 studies that described interventions aimed at improving timeliness. Most studies were performed in European Union member countries, including 24 studies performed in Great Britain and Ireland. Median times to diagnosis (range 8-60 days) and times to treatment (range 30-84 days) often exceeded published recommendations. Three studies found that timely care was associated with better survival, eight found no association and four reported better survival in patients who received less timely care. Interventions that improved timeliness included nurse-led care coordination, multidisciplinary meetings via teleconference and a standardised expedited "two-stop" diagnostic process.

Conclusions: Times to diagnosis and treatment of lung cancer are often longer than recommended. Factors associated with timeliness have been incompletely examined, and it remains unclear whether more timely care improves outcomes.

The care of patients with lung cancer requires complex coordination of services by medical and surgical specialists, healthcare administrators and social service providers. At the same time that health care in general is shifting towards more outpatient care, with fewer services requiring inpatient hospital stays, the complexity of lung cancer care has increased as more diagnostic and treatment options become available. The traditional approach of referring patients for consultation with multiple specialists in a sequential fashion often results in care that is perceived as slow, fragmented and poorly coordinated.

A number of guidelines and initiatives have been published to establish standards for timely care for patients with known or suspected lung cancer. These guidelines are primarily based on clinical opinion and relatively little has been published on the effects of these guidelines on processes of care.
In 1998 the British Thoracic Society (BTS) published specific recommended maximum time intervals for diagnosis and treatment in patients with lung cancer. ${ }^{1}$ In 2000 the UK National Health Service Cancer Plan provided goals for providing timely care to patients with all types of cancer. ${ }^{2}$ In 2000 the RAND Corporation also published quality indicators for timely diagnosis and treatment, focusing on the time from the first abnormal radiograph to diagnosis and from diagnosis to treatment. ${ }^{3}$ In 2003 the American College of Chest Physicians provided recommendations for the practice organisation of lung cancer care in the USA, with an emphasis on integrated multidisciplinary care. ${ }^{4}$ While timely care may contribute substantially to patients' quality of life and emotional well-being, it remains unclear whether timely care also improves patient outcomes.

In this systematic review we aimed to summarise all recently published studies that described the timeliness of care in patients with lung cancer, identified factors that were associated with more or less timely care, or examined the association between the timeliness of care and lung cancer outcomes, including stage distribution and survival. In addition, we aimed to identify studies that evaluated interventions to improve the timeliness of care for patients with lung cancer.

\section{METHODS}

Systematic methods were used to identify studies, assess eligibility for inclusion, evaluate quality, abstract data and synthesise results.

\section{Search strategy and selection criteria}

We searched MEDLINE to identify studies published between 1 January 1995 and 1 June 2007. Studies published before 1995 were excluded because they were included in a previous review ${ }^{5}$ and may be less relevant to current healthcare practices. We developed three separate search strategies to capture relevant studies that (1) described the timeliness of care in patients with lung cancer, (2) examined the association between timeliness of care and lung cancer outcomes and (3) described an intervention aimed at improving timeliness of lung cancer care (see Appendix). In addition, we manually searched reference lists of included studies and review articles and reviewed practice guidelines and systematic reviews. We included human studies published in the English language and excluded studies published only as abstracts.

All studies that included patients with known or suspected lung cancer were considered, including studies reporting patterns of care for patients with 


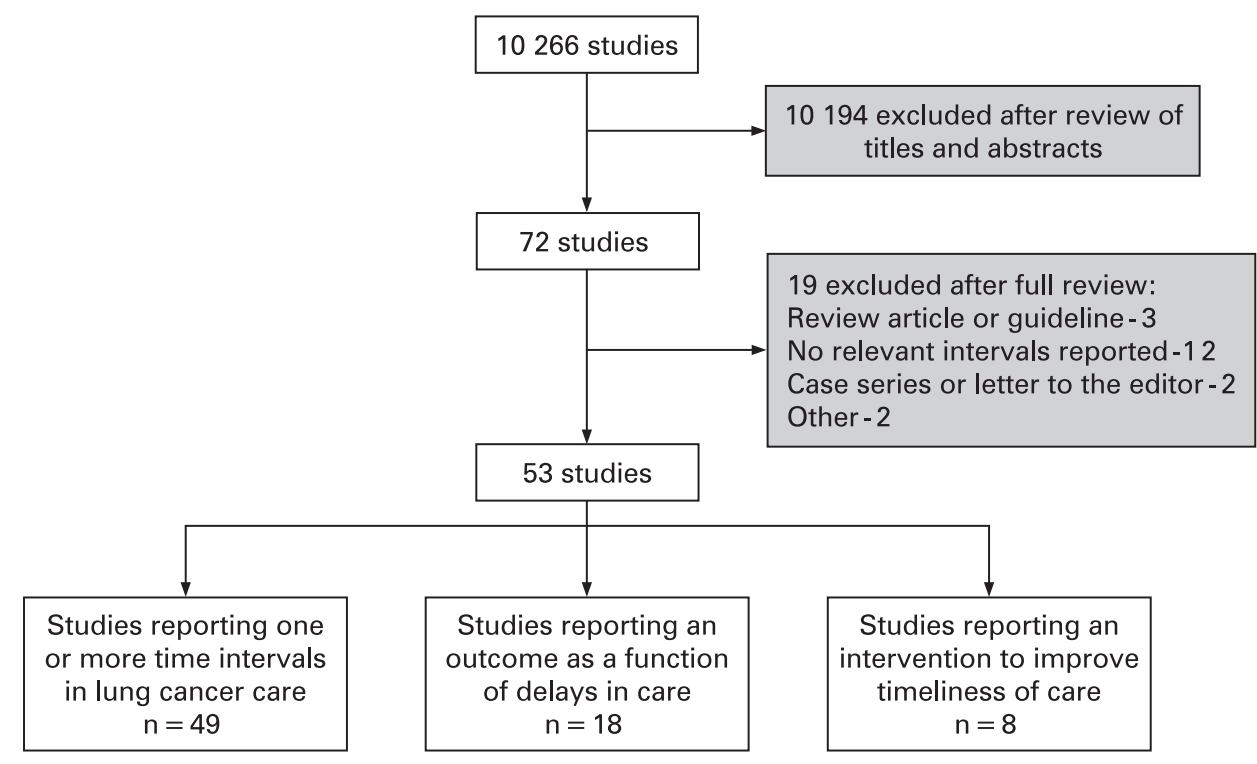

Figure 1 Search strategy results and study exclusions. Search strategies yielded a total of 10266 unique studies, of which 53 are included in this review. Included studies were grouped into three overlapping categories based on reported results.

symptoms that could be suggestive of lung cancer or patients with abnormal imaging test results.

\section{Data abstraction and quality assessment}

One investigator (JKO) reviewed all titles and abstracts and excluded articles that were obviously irrelevant or contained no primary data. Additional studies were excluded after review of the full article. Two investigators (JKO and EMS) assessed quality and abstracted the following data elements from included studies: study design, patient population, sample size, relevant time intervals and outcomes. Disagreements were resolved by discussion and by consultation with the senior investigator.

For studies of timeliness of care to be included we required that (1) the study reported one or more time intervals in the process of lung cancer diagnosis and treatment and (2) at least $75 \%$ of patients were diagnosed with lung cancer or results for patients with lung cancer were reported separately. For studies that examined the association between timeliness and lung cancer outcomes, we also required that the study reported overall and/or disease-free survival, stage distribution, tumour size or resectability in relation to timeliness of care, in addition to criteria (1) and (2) listed above.

Studies of interventions were included if they described any intervention aimed at improving the timeliness of care for patients with lung cancer, provided that they met the inclusion criteria that we used for studies of timeliness. We did not include studies that described a multidisciplinary team meeting, or tumour board, as this approach has already been widely adopted in cancer care. Studies of multidisciplinary clinics or dedicated lung mass clinics were included.

\section{Data synthesis}

When available from published studies, we report the median and range or interquartile range (IOR) for relevant time intervals in lung cancer care. Mean time intervals were reported only when no other data were available from published studies. Heterogeneity in study methods and reporting of results precluded quantitative synthesis.

\section{RESULTS}

Our search yielded 10266 potentially relevant studies. We excluded 10194 studies during the initial review, leaving 72 studies for further detailed review (fig 1). Nineteen studies were excluded after a full review and therefore 53 studies were included in the final analysis (see table in online supplement).

A total of 49 studies described at least one time interval in lung cancer care. Eighteen studies examined the association between timeliness and one or more outcomes, including 15 studies that examined survival, ${ }^{6-20} 4$ studies that examined stage distribution ${ }^{11} 162122$ and 1 that examined tumour growth. ${ }^{23}$ Eight studies that described an intervention to improve timeliness of care were identified. ${ }^{24-31}$

The majority of studies were performed in European Union member countries, including 24 studies performed in Great Britain and Ireland. Only five studies were performed in the USA, including one large study from Hawaii, ${ }^{32}$ one small study from Massachusetts ${ }^{33}$ and three studies conducted in US Veterans Affairs (VA) facilities. ${ }^{132631}$ Four Canadian studies $^{2134-36}$ and four Japanese studies ${ }^{12} 151637$ were identified, including several studies that examined delays in care after mass population-based screening. We also included two studies from Turkey, ${ }^{17}{ }^{38}$ one study from Australia ${ }^{24}$ and one study from Malaysia. ${ }^{9}$

Overall, study quality was uneven (fig 2). A total of 11 population-based reports were identified, ${ }^{6} 18193436$ 38-43 while most studies described care provided at tertiary care referral centres. Only nine studies enrolled patients prospectively. ${ }^{17} 2324303944-47$ While many studies were limited by small sample sizes, eight studies included more than 1000 patients with lung cancer. ${ }^{11} 14293234-3643$ In several studies basic demographic information was not reported, nor was information about histology or stage distribution. Information about the statistical analysis was often incomplete (fig 2).

\section{Timeliness of lung cancer care}

Forty-nine studies described at least one interval in the continuum from symptom onset to initial treatment. Reported median times from the primary care visit to referral for specialist evaluation were 13 days (range $0-21$ days), ${ }^{48}$ 
Studies of timeliness $(n=49)$

Cohort assembly

Were patients enrolled prospectively?

Was the study population-based?

Clinical characteristics

Did the study report patient age and gender?

Did the study report information about treatment?

Were inclusion and exclusion criteria clearly defined?

Statistical analysis

Did the study report number of patients in each analysis?

Did the study report a multivariate analysis?*

Did the study report how incomplete data was handled?

Studies of timeliness and outcome $(n=18)$

Was outcome specifically defined?

Was outcome stratified by NSCLC vs SCLC

Did the study report a multivariate analysis?

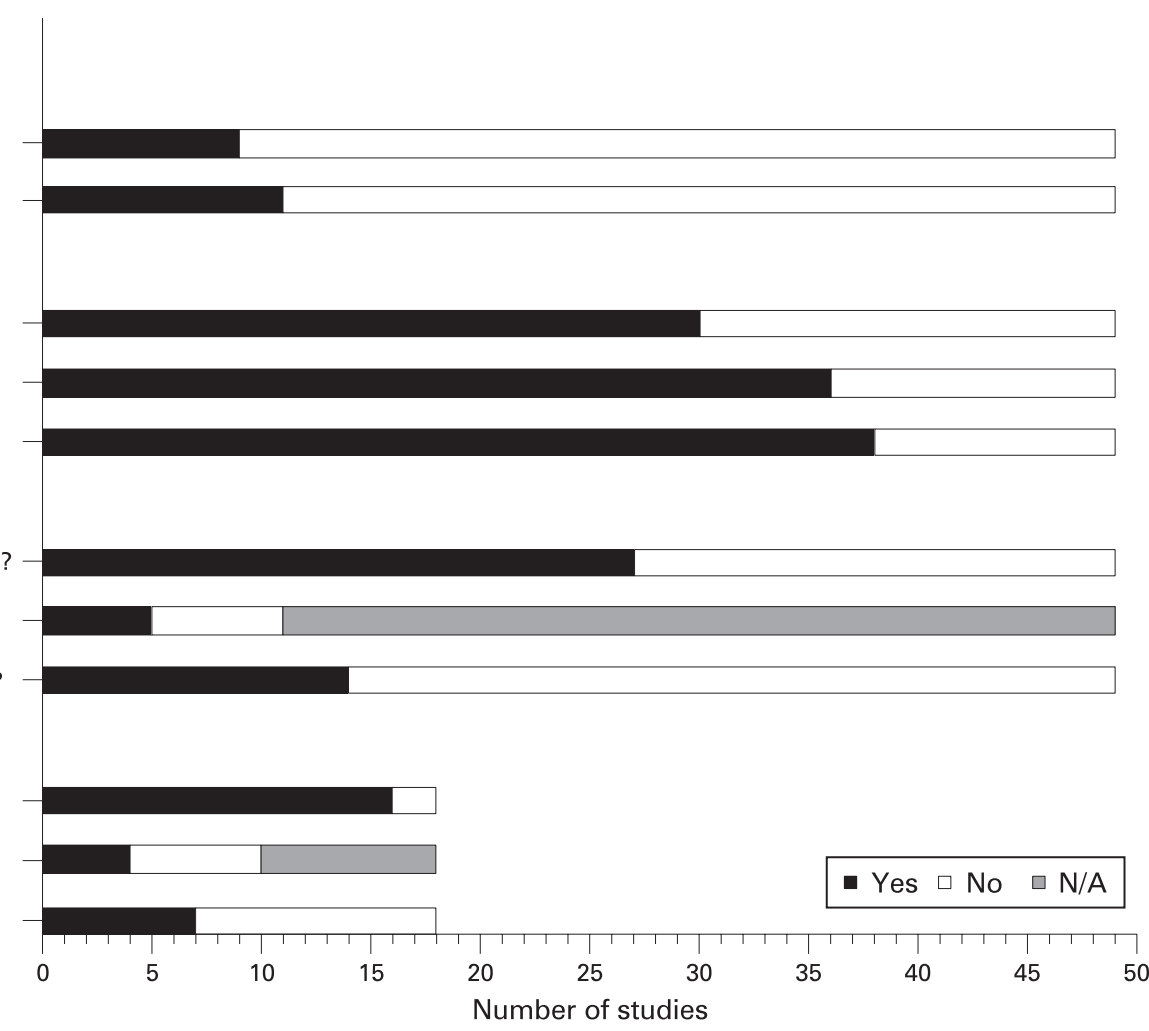

Figure 2 Study quality. Top: Number of studies that met each quality indicator for studies that described timeliness of care. Bottom: Studies that reported an analysis of clinical outcomes as a function of timeliness of care were assessed using additional quality criteria. ${ }^{*}$ If the study examined predictors of timely care, did the authors report a multivariate analysis to control for differences between groups? NSCLC, non-small cell lung cancer; SCLC, small cell lung cancer.

16 days, $^{6} 33$ days (IOR 12-68 days) ${ }^{49}$ and 33 days (range 0 477 days). ${ }^{39}$ Two studies demonstrated that patients often made multiple visits to their primary care physician before additional investigation were initiated. ${ }^{50} 51$ This was particularly evident in patients with superior sulcus tumours, who were often treated for presumed musculoskeletal shoulder pain before further evaluation took place. ${ }^{37} 42$

Adherence with existing guidelines for timeliness of lung cancer care was modest (table 1 ). In the eight studies reporting time from referral to first respiratory specialist visit, median times were all within the 14 days recommended by the NHS Cancer Care Plan, but seven studies reported a median time that exceeded the BTS recommendation for a maximum time of 7 days. $^{6} 101920294155$ Both guidelines have set target intervals for the maximum wait for all patients rather than median wait times. Three studies reported median times from specialist consultation to surgery that exceeded the 56-day maximum recommended by the BTS. ${ }^{21} 2654$ The most commonly cited reasons for delay were the need for multiple diagnostic tests and consultations.

The NHS National Cancer Plan targets a maximum interval of 1 month from diagnosis to treatment. ${ }^{2}$ In 5 of 11 studies the median time from diagnosis to treatment exceeded this recommendation and in several studies the median time approached or exceeded the 6-week maximum recommended by the RAND Corporation (table 1 ). ${ }^{3}$

Median times from diagnosis to treatment were similar across geographical regions (fig 3). In general, longer median times from diagnosis to treatment were reported in studies that limited analysis to patients treated with surgery or radiotherapy, which also tended to have larger sample sizes.

\section{Predictors of timely care}

Fourteen studies reported an analysis that identified predictors of timely care or provided descriptive information about reasons for delays in care (table 2).671718 ${ }^{64-36} 42454649525457$ Many studies examined the effect of age on timeliness of care, but the results were mixed. ${ }^{6}{ }^{17} 18$ 34-36 Patients with early-stage cancer often waited longer for a diagnosis, probably due to the need for multiple diagnostic tests to achieve a diagnosis. ${ }^{56}$ One Canadian study reported a tendency towards admitting patients with limited financial resources to the hospital, thereby expediting their care. ${ }^{34}$ Factors associated with less timely care included atypical symptoms, co-morbid conditions, teaching hospital setting, receipt of curative (versus palliative) radiotherapy, initial referral to a non-respiratory physician, requirement for multiple diagnostic tests and care received at more than one healthcare facility. Household income, ${ }^{34-36} 52$ gender, ${ }^{35}{ }^{36}$ hospital volume, ${ }^{35}$ rural residence ${ }^{35}$ and distance travelled to obtain care $^{3652}$ were not associated with timeliness.

\section{Timeliness of lung cancer care and outcomes}

A total of 18 studies examined the association between timely care and patient outcomes. Fifteen studies looked at the effect of timeliness on survival and reported mixed results, including eight studies that showed no association between timeliness and outcome. ${ }^{6-13}$

Three studies demonstrated worse survival in patients with delayed diagnosis and treatment, including two studies that included patients identified through population-based mass screening. ${ }^{14-16}$ Kashiwabara et al reported both an analysis of patients with tumours that were initially missed on screening but identified 1 year later on repeat screening, and an analysis of 


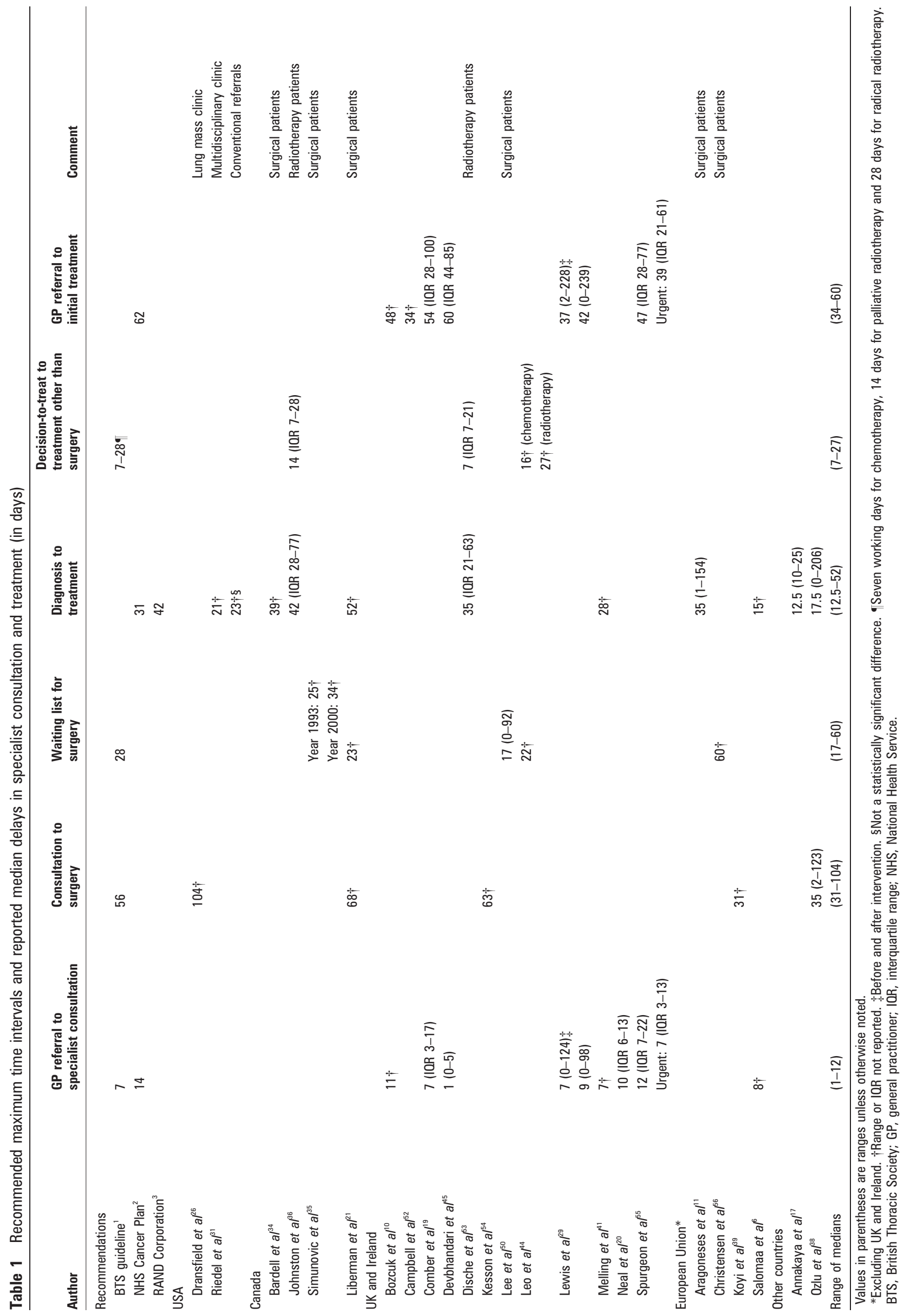




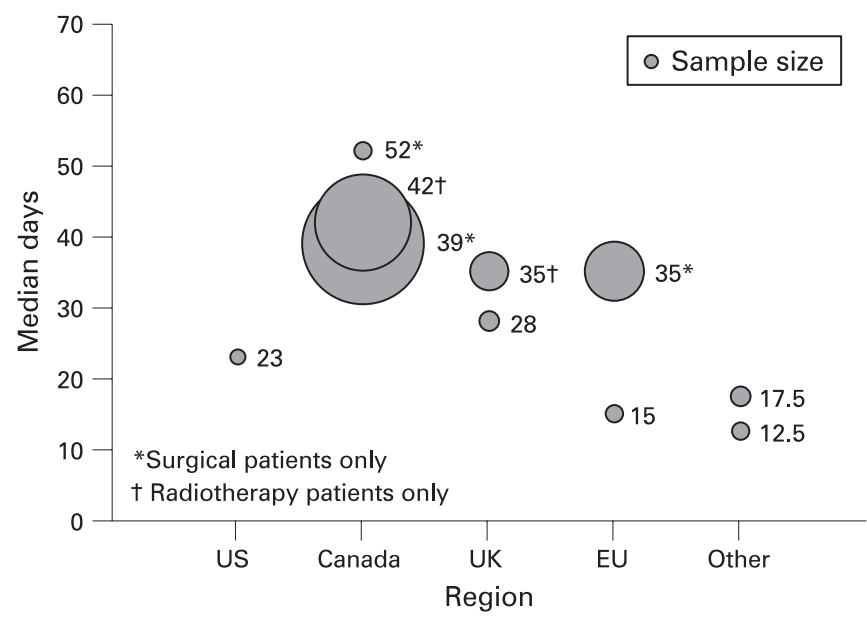

Figure 3 Distribution of median times from diagnosis to treatment. Sample size, patient cohort and reported median time from diagnosis to treatment varied widely among studies that reported this interval. Labels indicate reported median values for time from diagnosis to treatment. Bubbles indicate relative sample size, which ranged from 89 to 4366 patients. EU, European Union (excluding UK and Ireland); UK, United Kingdom and Ireland; US, United States.

patients with correctly identified abnormalities who did not attend follow-up with a physician. ${ }^{12}{ }^{16}$ Median survival was worse in those who did not follow up promptly and correlated with increases in tumour size. The hazard of death increased by $5.5 \%$ for each additional $1 \mathrm{~mm}$ in tumour size at the time of diagnosis. ${ }^{16}$ In another study of patients with lung cancer identified on a screening chest radiograph, Kanashiki et al found that survival was worse in patients who received a diagnosis more than 4 months after the initial abnormal chest radiograph. ${ }^{15}$ Buccheri et al also observed worse survival among patients with longer time from symptom onset to specialist referral. ${ }^{14}$

Paradoxically, four studies showed better median survival for patients who received less timely care. ${ }^{17-20}$ Annakkaya et al reported a statistically significant difference in survival when comparing patients with a symptom-to-diagnosis interval above and below 60 days, but not 45 or 90 days. ${ }^{17}$ Comber et al reported better lung cancer-specific survival in patients with longer times to treatment compared with those who waited less than 1 month. ${ }^{19}$ In the study by Myrdal et al, patients with more advanced disease experienced significantly shorter times to diagnosis and treatment. ${ }^{18}$ The authors reported a significant interaction between tumour stage and timeliness, suggesting that the increased mortality in patients with shorter time intervals was due to more advanced disease at the time of presentation. Similarly, Neal et al reported that survival was worse in patients with lung cancer who had urgent versus nonurgent referrals, and that patients with urgent referrals had more advanced stage disease at diagnosis. ${ }^{20}$

Four studies examined the effect of timeliness on stage. Three studies showed no effect, ${ }^{112122}$ while one study demonstrated an association between longer delays and more advanced stage. ${ }^{16}$ Liberman et al limited their analysis to surgically treated patients in Canada and found no effect of preoperative delay on pathological stage. ${ }^{21}$

One study examined the relationship between tumour growth and delays in initiation of radiotherapy in 29 patients with non-small cell lung cancer in the UK. ${ }^{23}$ Comparing tumours seen on diagnostic and planning CT scans separated by a median interval of 54 days, the median increase in tumour cross-sectional area was $19 \%$ but tumour size increased by as much as $373 \%$ in one patient. Six patients became ineligible for curative treatment, all of whom were diagnosed with squamous cell carcinomas. This study supports the notion that tumour cell biology may be a determinant of the impact of delay on patient outcome, as all of the patients with adenocarcinomas were able to proceed with treatment as planned.

\section{Improving timeliness of lung cancer care}

We identified eight studies that described an intervention to improve timeliness of care and/or surgical resection rates. ${ }^{24-31}$ The studies evaluated different approaches to improving timeliness of care, including multidisciplinary clinics, nurse-led care coordination, telemedicine and a novel "two-stop" outpatient diagnostic process.

Table 2 Variables associated with less timely care in lung cancer

\begin{tabular}{|c|c|}
\hline Variable & Effect size \\
\hline Younger age $^{34}$ & $\begin{array}{l}\text { Patients aged }>70 \text { years were } 9 \% \text { more likely to have surgery within } 2 \text { weeks of diagnosis } \\
\text { than patients age }<70 \text { years (OR } 1.09,95 \% \mathrm{Cl} 1.01 \text { to } 1.17)^{*}\end{array}$ \\
\hline Teaching hospital $^{34}$ & $\begin{array}{l}\text { Patients were } 30 \% \text { less likely to have surgery within } 2 \text { weeks of diagnosis in a teaching } \\
\text { hospital than in a non-teaching hospital (OR } 0.70,95 \% \mathrm{Cl} 0.66 \text { to } 0.74)^{*}\end{array}$ \\
\hline Curative vs palliative radiotherapy ${ }^{36}$ & $\begin{array}{l}\text { Median 28-day wait for radiotherapy for distant disease compared with median 49-day } \\
\text { wait for radiotherapy for limited disease } \dagger\end{array}$ \\
\hline $\begin{array}{l}\text { Initial referral to a non-respiratory } \\
\text { physician }^{54}\end{array}$ & $\begin{array}{l}\text { Median 35-day wait for bronchoscopy for patients initially referred to a non-respiratory } \\
\text { physician compared with median 7-day wait for all patients }\end{array}$ \\
\hline $\begin{array}{l}\text { Increasing number of diagnostic tests } \\
\text { needed to achieve a diagnosis }\end{array}$ & $\begin{array}{l}\text { Median GP referral to treatment interval was } 44 \text { days (range } 37-60 \text { ) for patients with } \\
\text { diagnosis established on initial bronchoscopy compared with } 80 \text { days (range } 54-107) \text { for } \\
\text { patients requiring additional procedures }(p<0.01) \uparrow^{45}\end{array}$ \\
\hline $\begin{array}{l}\text { Multiple hospitals required to obtain } \\
\text { appropriate diagnostic tests and/or } \\
\text { treatment }^{34} 57\end{array}$ & $\begin{array}{l}\text { Mean wait time } 31.6 \text { days for patients requiring transfer to another hospital for treatment } \\
\text { compared with } 25.9 \text { days for patients diagnosed and treated at the same facility }\end{array}$ \\
\hline Age $>70$ years $^{35}$ & $\begin{array}{l}\text { Patients aged }>70 \text { years had a } 13 \% \text { increase in median wait time for surgery compared } \\
\text { with younger patients } \dagger\end{array}$ \\
\hline$\geqslant 1$ co-morbidity ${ }^{1735}$ & $\begin{array}{l}\text { Patients with } \geqslant 1 \text { co-morbidity had a } 7 \% \text { increase in median wait time to surgery compared } \\
\text { with patients without co-morbidities } \dagger\end{array}$ \\
\hline Atypical symptoms at presentation ${ }^{42} 49$ & $\begin{array}{l}\text { Median } 29 \text { days for patients presenting with respiratory symptoms versus } 104 \text { days for } \\
\text { patients with non-specific symptoms }{ }^{49} \\
\text { Median time from onset of pain to diagnosis was } 150 \text { days for patients with superior } \\
\text { sulcus tumours }{ }^{42}\end{array}$ \\
\hline
\end{tabular}

*Multivariate analysis. †Univariate analysis. 
Three studies that evaluated patient care coordination through a dedicated lung mass clinic or a multidisciplinary clinic did not show a reduction in delays with either approach. ${ }^{24}{ }^{26}{ }^{31}$ In contrast, Davison et al reported a successful effort to improve communication between specialists and participation by thoracic surgeons in the UK by using telemedicine multidisciplinary meetings in the care of patients with suspected lung cancer. ${ }^{25}$ Leary and Corrigan reported a significant improvement in timeliness with a nurse-led intervention that included a detailed analysis of the local care process, restructuring of referral patterns and hospital-wide educational initiatives. ${ }^{28}$ Lewis et al performed a retrospective study of 1044 patients with suspected lung cancer referred to a single facility during the 12 months before and 24 months after introduction of the UK Department of Health urgent referral guidelines. ${ }^{29}$ The authors found that the median time from referral to first visit with a respiratory specialist increased from 7 to 9 days. The authors concluded that the "2-week wait" initiative did not achieve the primary objective of reducing waiting times for lung cancer care.

Two studies reported reductions in time to treatment using a "two-stop" diagnostic process for expediting investigation of patients with suspected lung cancer. Laroche et al described 275 patients treated at a single tertiary care facility in the UK. ${ }^{27}$ Patients received CT, biopsy and/or other diagnostic tests at the initial visit and a treatment plan was developed during a multidisciplinary meeting within 3 days. The median time from first specialist visit to surgery was reduced by $50 \%$, and the overall successful surgical resection rate was $25 \%$. Murray et al subsequently randomised 88 patients with suspected lung cancer to undergo investigation and treatment via a centralised "two-stop" diagnostic process or conventional diagnosis at the local hospital..$^{30}$ Time from first presentation to treatment was 3 weeks in the centralised arm compared with 7 weeks in the conventional arm.

\section{DISCUSSION}

Despite the increasing emphasis on efficient and timely delivery of health care, we found that reported times to diagnosis and treatment for patients with lung cancer in a variety of different healthcare settings are often longer than recommended by published guidelines.

In a previous review, Jensen and colleagues identified 16 studies published before 2001 that reported delays in lung cancer management. ${ }^{5}$ Like our study, they found considerable heterogeneity in study design, patient population and interval definitions, limiting comparisons across studies. They found very few published data on health system delays and little evidence that delays were associated with survival or stage. ${ }^{5}$

In this systematic review, limited conclusions can be drawn from the studies of timeliness of care and outcomes which revealed mixed and even paradoxical results. Studies were limited by referral centre bias and by inadequate methods for dealing with confounding variables such as age, stage, tumour histology, co-morbidities and the presence of symptoms at presentation. Most large studies were performed by using administrative databases with only surgical patients and included limited clinical information about tumour size, comorbidities and adjuvant therapy. It is possible that some of the non-surgically treated patients excluded from these studies would have been eligible for surgical treatment had a diagnosis been made sooner. Smaller studies often included all patients with lung cancer and included more detailed clinical information, but typically were underpowered and often did not adjust for variables that might confound the relationship between timeliness and survival. Such confounding may explain the paradoxical results reported in several studies in which patients with the most timely care had the worst survival rates. In these studies, patients with advanced disease at the time of presentation are more likely to have symptoms and signs of malignancy and may be more likely to receive prompt diagnosis and palliative treatment due to earlier specialist referral and fewer diagnostic tests needed to achieve a diagnosis. These patients may also be more likely to receive supportive care only, and to die earlier than patients with early-stage disease. Additionally, some studies were potentially limited by lead time bias, especially studies that measured survival from the start of treatment.

Several studies examined ways to improve timeliness of care for patients with lung cancer. While the multidisciplinary clinic approach to care has been encouraged as a way to improve both timeliness and quality of care, there is little evidence that this approach actually improves timeliness of lung cancer care. Studies do show that multidisciplinary clinics are associated with increased rates of active treatment, including surgical resection and chemotherapy, and may therefore provide better quality medical care. The "two-stop" diagnostic process described by Laroche et al and Murray et al appears effective in significantly reducing diagnostic delay. The main limitation to this approach is that it requires a high level of care coordination by multiple specialty services. Lewis et al concluded that the UK "two-week wait" initiative failed to reduce wait times because the increase in urgent referrals burdened specialist services, leading to longer waits for patients referred non-urgently.

Our study has several limitations. We limited our literature search to a single computerised database and included only studies published in the English language. However, our MEDLINE search was comprehensive, and we also identified studies by scanning reference lists from included studies and recent reviews. Most of the studies were performed in European Union member countries, limiting conclusions that one can make about lung cancer care in non-European Union healthcare settings. In particular, only five studies were performed in the USA, including three carried out in VA hospitals. Direct comparison of timeliness in different countries and healthcare systems may be misleading as patterns of care vary within different regions. However, we believe that such comparisons are still valuable, particularly given the paucity of recommendations that address timeliness of lung cancer care.

While heterogeneity in study methods precluded us from performing a quantitative synthesis, the observation that time intervals were often longer than recommended in numerous and varied healthcare settings strongly supports the generalisability of our findings. However, we believe that future studies would benefit by using more standardised methods. In particular, we recommend that a standard definition for each relevant time interval be accepted.

We need more studies that describe lung cancer care in private hospitals, teaching facilities, county hospitals and Health Maintenance Organizations in the USA as most of the US population receives care in such facilities. Prospective studies with consecutively enrolled patients would reduce confounding due to selection bias, recall bias, missing data and variations in treatment practices. Studies should also examine the effect of patient factors, including race and ethnicity, on the timeliness of care. 
While it remains unclear whether more timely care improves outcomes in lung cancer, improving timeliness is important independent of its ultimate effect on survival. Our patients expect, deserve and appreciate care that is timely, safe and effective.

Funding: This study was supported by the VA Office of Quality and Performance. Competing interests: None.

The views expressed in this paper are those of the authors and do not necessarily represent the views of the Department of Veterans Affairs.

Provenance and peer review: Not commissioned; externally peer reviewed.

\section{REFERENCES}

1. British Thoracic Society. BTS recommendations to respiratory physicians for organising the care of patients with lung cancer. The Lung Cancer Working Party of the British Thoracic Society Standards of Care Committee. Thorax 1998;53(Suppl 1): $\$ 1-8$.

2. Department of Health. The NHS cancer plan. London: Department of Health, 2000.

3. Reifel J. Lung cancer. In: Asch S, Kerr E, Hamilton E, et al., eds Quality of care for oncologic conditions and HIV: a review of the literature and quality indicators. RAND Corporation, 2000.

4. Alberts WM, Bepler G, Hazelton T, et al. Lung cancer. Practice organization. Chest 2003; 123:332-7S.

5. Jensen AR, Mainz J, Overgaard J. Impact of delay on diagnosis and treatment of primary lung cancer. Acta Oncol 2002;41:147-52.

6. Salomaa ER, Sallinen S, Hiekkanen H, et al. Delays in the diagnosis and treatment of lung cancer. Chest 2005;128:2282-8.

7. Ringbaek T, Borgeskov S, Lange $P$, et al. Diagnostic and therapeutic process and prognosis in suspected lung cancer. Scand Cardiovasc J 1999;33:337-43.

8. Pita-Fernandez S, Montero-Martinez C, Pertega-Diaz S, et al. Relationship between delayed diagnosis and the degree of invasion and survival in lung cancer. $J$ Clin Epidemiol 2003;56:820-5.

9. Loh LC, Chan LY, Tan RY, et al. Effect of time delay on survival in patients with nonsmall cell lung cancer-a Malaysian study. Asia Pac J Public Health 2006;18:69-71.

10. Bozcuk $\mathbf{H}$, Martin C. Does treatment delay affect survival in non-small cell lung cancer? A retrospective analysis from a single UK centre. Lung Cancer 2001;34:243-52.

11. Aragoneses FG, Moreno N, Leon P, et al. Influence of delays on survival in the surgical treatment of bronchogenic carcinoma. Lung Cancer 2002;36:59-63.

12. Kashiwabara K, Koshi S, Ota K, et al. Outcome in patients with lung cancer found retrospectively to have had evidence of disease on past lung cancer mass screening roentgenograms. Lung Cancer 2002;35:237-41.

13. Quarterman $\mathbf{R L}$, McMillan A, Ratcliffe $\mathrm{MB}$, et al. Effect of preoperative delay on prognosis for patients with early stage non-small cell lung cancer. J Thorac Cardiovasc Surg 2003;125:108-13.

14. Buccheri G, Ferrigno D. Lung cancer: clinical presentation and specialist referral time. Eur Respir J 2004;24:898-904.

15. Kanashiki M, Satoh H, Ishikawa $\mathrm{H}$, et al. Time from finding abnormality on massscreening to final diagnosis of lung cancer. Oncol Rep 2003;10:649-52.

16. Kashiwabara $\mathbf{K}$, Koshi S, Itonaga $\mathrm{K}$, et al. Outcome in patients with lung cancer found on lung cancer mass screening roentgenograms, but who did not subsequently consult a doctor. Lung Cancer 2003;40:67-72.

17. Annakkaya AN, Arbak P, Balbay 0 , et al. Effect of symptom-to-treatment interval on prognosis in lung cancer. Tumori 2007;93:61-7.

18. Myrdal G, Lambe M, Hillerdal G, et al. Effect of delays on prognosis in patients with non-small cell lung cancer. Thorax 2004;59:45-9.

19. Comber $\mathbf{H}$, Cronin DP, Deady S, et al. Delays in treatment in the cancer services: impact on cancer stage and survival. Ir Med J 2005;98:238-9.

20. Neal RD, Allgar VL, Ali N, et al. Stage, survival and delays in lung, colorectal, prostate and ovarian cancer: comparison between diagnostic routes. $\mathrm{Br} J \mathrm{Gen}$ Pract 2007; 57:212-9.

21. Liberman M, Liberman D, Sampalis JS, et al. Delays to surgery in non-small-cell lung cancer. Can J Surg 2006:49:31-6.

22. Billing JS, Wells FC. Delays in the diagnosis and surgical treatment of lung cancer. Thorax 1996:51:903-6.

23. O'Rourke N, Edwards R. Lung cancer treatment waiting times and tumour growth. Clin Oncol (R Coll Radiol) 2000;12:141-4.

24. Conron M, Phuah S, Steinfort D, et al. Analysis of multidisciplinary lung cancer practice. Intern Med J 2007;37:18-25.

25. Davison AG, Eraut CD, Haque AS, et al. Telemedicine for multidisciplinary lung cancer meetings. J Telemed Telecare 2004;10:140-3.

26. Dransfield MT, Lock BJ, Garver RI Jr. Improving the lung cancer resection rate in the US Department of Veterans Affairs Health System. Clin Lung Cancer 2006; 7:268-72.

27. Laroche $\mathbf{C}$, Wells $F$, Coulden $\mathrm{R}$, et al. Improving surgical resection rate in lung cancer. Thorax 1998;53:445-9.

28. Leary A, Corrigan P. Redesign of thoracic surgical services within a cancer networkusing an oncology focus to inform change. Eur J Oncol Nurs 2005;9:74-8.
29. Lewis NR, Le Jeune I, Baldwin DR. Underutilisation of the 2-week wait initiative for lung cancer by primary care and its effect on the urgent referral pathway. $\mathrm{Br} \mathrm{J}$ Cancer 2005;93:905-8.

30. Murray PV, O'Brien ME, Sayer R, et al. The pathway study: results of a pilot feasibility study in patients suspected of having lung carcinoma investigated in a conventional chest clinic setting compared to a centralised two-stop pathway. Lung Cancer 2003:42:283-90.

31. Riedel RF, Wang $X$, McCormack $M$, et al. Impact of a multidisciplinary thoracic oncology clinic on the timeliness of care. J Thorac Oncol 2006;1:692-6.

32. Liu DM, Kwee SA. Demographic, treatment, and survival patterns for Native Hawaiians with lung cancer treated at a community medical center from 1995 to 2001. Pac Health Dialog 2004:11:139-45.

33. Finlay GA, Joseph $B$, Rodrigues CR, et al. Advanced presentation of lung cancer in Asian immigrants: a case-control study. Chest 2002;122:1938-43.

34. Bardell T, Belliveau P, Kong W, et al. Waiting times for cancer surgery in Ontario: 1984-2000. Clin Oncol (R Coll Radiol) 2006;18:401-9.

35. Simunovic $\mathbf{M}$, Theriault ME, Paszat $L$, et al. Using administrative databases to measure waiting times for patients undergoing major cancer surgery in Ontario, 1993-2000. Can J Surg 2005;48:137-42.

36. Johnston GM, MacGarvie VL, Elliott D, et al. Radiotherapy wait times for patients with a diagnosis of invasive cancer, 1992-2000. Clin Invest Med 2004:27:142-56.

37. Ichinohe K, Takahashi M, Tooyama N. Delay by patients and doctors in treatment of Pancoast tumor. Wien Klin Wochenschr 2006;118:405-10.

38. Ozlu T, Bulbul Y, Oztuna F, et al. Time course from first symptom to the treatment of lung cancer in the Eastern Black Sea Region of Turkey. Med Princ Pract 2004;13:211-4.

39. Koyi H, Hillerdal G, Branden E. Patient's and doctors' delays in the diagnosis of chest tumors. Lung Cancer 2002:35:53-7.

40. Mansson J, Bjorkelund C, Hultborn R. Symptom pattern and diagnostic work-up of malignancy at first symptom presentation as related to level of care. A retrospective study from the primary health care centre area of Kungsbacka, Sweden. Neoplasma 1999:46:93-9.

41. Melling PP, Hatfield AC, Muers MF, et al. Lung cancer referral patterns in the forme Yorkshire region of the UK. Br J Cancer 2002;86:36-42.

42. Strojan P, Debevec M, Kovac V. Superior sulcus tumor (SST): management at the Institute of Oncology in Ljubljana, Slovenia, 1981-1994. Lung Cancer 1997;17:249-59.

43. Gregor A, Thomson CS, Brewster DH, et al. Management and survival of patients with lung cancer in Scotland diagnosed in 1995: results of a national population based study. Thorax 2001:56:212-7.

44. Leo $\mathbf{F}$, Venissac N, Poudenx $\mathbf{M}$, et al. Multidisciplinary management of lung cancer: how to test its efficacy? J Thorac Oncol 2007;2:69-72.

45. Devbhandari MP, Soon SY, Quennell P, et al. UK waiting time targets in lung cance treatment: are they achievable? Results of a prospective tracking study. J Cardiothorac Surg 2007;2:5.

46. Deegan PC, Heath L, Brunskill J, et al. Reducing waiting times in lung cancer. J R Coll Physicians Lond 1998;32:339-43.

47. Fergusson RJ, Gregor A, Dodds R, et al. Management of lung cancer in South East Scotland. Thorax 1996:51:569-74.

48. Lim WS, Macfarlane JT, Deegan PC, et al. How do general practitioners respond to reports of abnormal chest X-rays? J $R$ Soc Med 1999:92:446-9.

49. Bjerager $\mathbf{M}$, Palshof $T$, Dahl $R$, et al. Delay in diagnosis of lung cancer in general practice. Br J Gen Pract 2006;56:863-8.

50. Lee J, Marchbank A, Goldstraw P. Implementation of the British Thoracic Society recommendations for organising the care of patients with lung cancer: the surgeon's perspective. Ann R Coll Surg Engl 2002;84:304-8.

51. Corner J, Hopkinson J, Fitzsimmons D, et al. Is late diagnosis of lung cancer inevitable? Interview study of patients' recollections of symptoms before diagnosis. Thorax 2005;60:314-9.

52. Campbell NC, Elliott AM, Sharp $L$, et al. Impact of deprivation and rural residence on treatment of colorectal and lung cancer. Br J Cancer 2002;87:585-90.

53. Dische $\mathbf{S}$, Gibson D, Parmar M, et al. Time course from first symptom to treatment in patients with non-small cell lung cancer referred for radiotherapy: a report by the CHART Steering Committee. Thorax 1996;51:1262-5.

54. Kesson E, Bucknall CE, McAlpine LG, et al. Lung cancer-management and outcome in Glasgow, 1991-92. Br J Cancer 1998;78:1391-5.

55. Spurgeon P, Barwell F, Kerr D. Waiting times for cancer patients in England after general practitioners' referrals: retrospective national survey. $B M J$ 2000;320:838-9.

56. Christensen ED, Harvald $\mathrm{T}$, Jendresen $\mathrm{M}$, et al. The impact of delayed diagnosis of lung cancer on the stage at the time of operation. Eur J Cardiothorac Surg 1997; 12:880-4

57. Krishnasamy M, Wilkie E, Haviland J. Lung cancer health care needs assessment: patients' and informal carers' responses to a national mail questionnaire survey. Palliat Med 2001;15:213-27.

\section{APPENDIX: MEDLINE SEARCH STRATEGIES}

\section{Studies of timeliness of lung cancer care}

lung neoplasms/diagnosis[MESH] OR lung neoplasms/drug therapy[MESH] OR lung neoplasms/radiotherapy[MESH] OR lung neoplasms/surgery[MESH] OR lung neoplasms/therapy[MESH]

AND 
time factors[MeSH] OR waiting lists[MeSH] OR delay[text word] OR Timeliness[text word] OR Time[text word] OR prognosis[MESH]

AND 1995/01/01[PDat]: 2007/06/01[PDat] AND Humans[Mesh] AND English[lang]

\section{Studies of timeliness and outcome in lung cancer care}

lung neoplasms[MESH] OR lung cancer*[text word]

AND

time factors[MeSH] OR waiting lists[MeSH] OR delay[text word] OR timeliness[text word]) AND mortality[MeSH Terms] OR survival rate[MESH] OR survival analysis[MESH] OR survival[MeSH Terms]

AND 1995/01/01[PDat]: 2007/06/01[PDat] AND Humans[Mesh] AND English[lang]
Studies aimed at improving timeliness of lung cancer care lung neoplasms[MeSH] OR lung cancer*[text word]

AND

delivery of health care[MESH] OR program development[MESH] OR management information systems[MESH] OR outcome and process assessment (health care) [MESH] OR referral and consultation[MESH] OR practice management[MESH] AND

time factors[MeSH] OR waiting lists[MeSH] OR delay[text word] OR timeliness[text word] OR time[text word]

AND 1995/01/01[PDat]: 2007/06/01[PDat] AND Humans[Mesh] AND English[lang]

\section{Pulmonary puzzle}

\section{A 63-year-old male with marked eosinophilia and dyspnoea on exertion}

\section{CLINICAL PRESENTATION}

A 63-year-old male farmer presented marked eosinophilia. He had no symptoms of fever, night sweats or weight loss and no signs of anaemia, jaundice or lymphadenopathy. The leucocyte count was $17.27 \times 10^{9} / 1$, with $53.1 \%$ eosinophils, but no eggs or parasites were found in his faeces. Blood chemistry results were within normal limits except for an elevated alanine aminotransferase level of $77 \mathrm{U} / \mathrm{l}$. Serum total immunoglobulin E ( $\mathrm{IgE}$ ) was high at $340 \mathrm{kU} / \mathrm{l}$. Specific IgG antibodies to Taenia solium, Paragonimus westermani, Sparganum mansoni and Clonorchis sinensis were negative. The patient's first absolute eosinophil count during his hospitalisation was $27.25 \times 10^{9} / 1$. Both a chest CT scan and an echocardiograph were normal. A contrastenhanced CT scan of the liver showed multiple, small, illdefined, round, low-attenuating nodules with hepatomegaly. There was no evidence of lymphadenopathy on both a chest and abdominal CT scan. Bone marrow specimens revealed normocellular marrow with marked eosinophilia and no evidence of eosinophilic leukaemia. His eosinophils had increased to $46.32 \times 10^{9} / 1$, so he was treated with prednisolone at $1 \mathrm{mg} / \mathrm{kg} / \mathrm{day}$. The pronounced eosinophilia improved and he was discharged. We tapered the dose of prednisolone to $0.8 \mathrm{mg} /$ $\mathrm{kg} /$ day over 3 months and his eosinophil counts returned to normal at $0.02 \times 10^{9} / 1$

Four months later, he was admitted again with dyspnoea on exertion and hypoxaemia. The lower lungs exhibited fine bilateral crackles. The leucocyte count was $13.39 \times 10^{9} / 1$ with $0.7 \%$ eosinophils $\left(0.10 \times 10^{9} / 1\right)$. A chest radiograph showed peripheral reticulonodular opacities in both lungs. A highresolution $\mathrm{CT}$ showed fine reticulation and irregular linear opacity with predominant subpleural distribution (fig 1). Pulmonary function tests showed a reduction in diffusing capacity (55\% predicted)

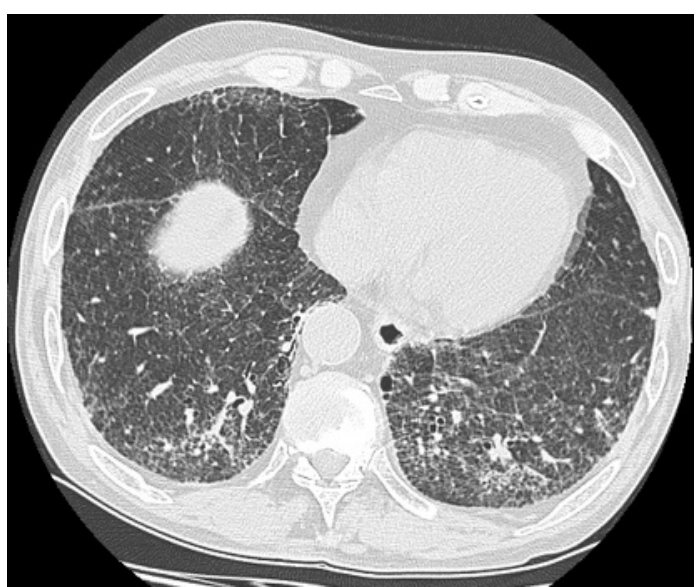

Figure 1 High-resolution CT through the lower lung zone on 11 February 2005. The scan shows bilateral irregular linear opacity with predominant subpleural distribution.

\section{QUESTION}

What questions might you ask the farmer?

See page 777 for the answer.

\section{S-H Kim, ${ }^{1}$ Y Nawa, ${ }^{2}$ H Y Kim, ${ }^{1}$ W Kwon, ${ }^{3}$ S J Yong, ${ }^{1}$ S H Jung ${ }^{4}$}

${ }^{1}$ Department of Internal Medicine, Yonsei University Wonju College of Medicine, Wonju, Korea; ${ }^{2}$ Division of Parasitology, Department of Infectious Diseases, Faculty of Medicine, University of Miyazaki, Kiyotake, Miyazaki, Japan; ${ }^{3}$ Department of Diagnostic Radiology, Yonsei University Wonju College of Medicine, Wonju, Korea;

${ }^{4}$ Department of Pathology, Yonsei University Wonju College of Medicine, Wonju, Korea

Correspondence to: Dr S H Jung, Department of Pathology, Yonsei University Wonju College of Medicine, Wonju, Korea; soonheej@yonsei.ac.kr

Competing interests: None.

Patient consent: Obtained.

Provenance and peer review: Not commissioned; externally peer reviewed. Thorax 2009;64:756. doi:10.1136/thx.2008.101352 